\title{
Estudios de grado, master y doctorado en Comunicación en España tras los 50 años de su instauración
}

\section{Bachelor, master, and PhD studies in Communication in Spain $\mathbf{5 0}$ years after their establishment}

\author{
Alejandro Álvarez-Nobell; Antonio Castillo-Esparcia; Isabel Ruiz-Mora
}

Cómo citar este artículo:

Álvarez-Nobell, Alejandro; Castillo-Esparcia, Antonio; Ruiz-Mora, Isabel (2022). "Estudios de grado, master y doctorado en Comunicación en España tras los 50 años de su instauración". Profesional de la información, v. 31, n. 1, e310109.

https://doi.org/10.3145/epi.2022.ene.09

Artículo recibido el 10-07-2021

Aceptación definitiva: 12-09-2021

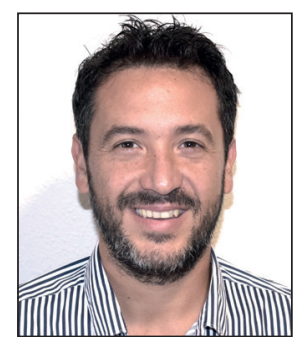

Alejandro Álvarez-Nobell

https://orcid.org/0000-0003-1384-3544

Universidad de Málaga

Facultad de Ciencias de la Comunicación Departamento de Comunicación

Audiovisual y Publicidad

León Tolstoi, s/n. 29071 Málaga, España

aan@uma.es

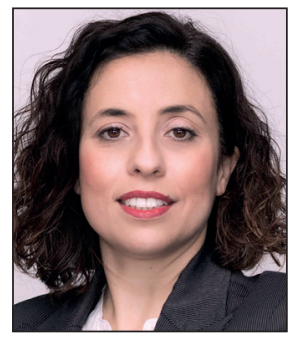

Isabel Ruiz-Mora $\triangle$

https://orcid.org/0000-0003-0723-9850

Universidad de Málaga

Facultad de Ciencias de la Comunicación

Departamento de Comunicación

Audiovisual y Publicidad

León Tolstoi, s/n. 29071 Málaga, España

isabelruiz@uma.es

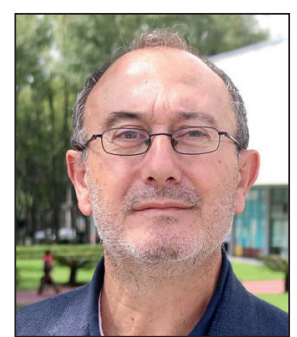

Antonio Castillo-Esparcia

https://orcid.org/0000-0002-9751-8628

Universidad de Málaga

Facultad de Ciencias de la Comunicación

Departamento de Comunicación

Audiovisual y Publicidad

León Tolstoi, s/n. 29071 Málaga, España

acastilloe@uma.es

\section{Resumen}

Los estudios de Comunicación en España se consolidan como resultado de la evolución experimentada en 50 años. Se pretende mostrar una radiografía que permita conocer el estado actual de la educación superior española en esta materia. Para ello se ha realizado un análisis de la estructura y composición de los grados y posgrados (masters oficiales y doctorado) de Comunicación de las universidades públicas y privadas de España, prestando especial atención a los centros que acogen estos estudios, las personas que con su desempeño contribuyen a su funcionamiento, su oferta y distribución. Mediante un diseño metodológico exploratorio y descriptivo, a través de la técnica del análisis de contenido, se han revelado las variables de estudio a partir de la oferta e información disponible y accesible en las webs institucionales de las universidades que conforman el sistema español en esta materia. Se destaca la dificultad para acceder a los datos y la falta de criterios comunes (o cumplimiento de estos) para presentar la información sobre la oferta formativa oficial. Los resultados arrojan una mayor oferta formativa a nivel nacional, pero evidencian su dispersión en cuanto a tipo, oferta de plaza, tipo de universidad y distribución territorial. Destaca la brecha de género en los responsables de los centros y entre la plantilla de profesorado; y el fenómeno creciente de los grados dobles o combinados, sobre todo en los centros privados.

\section{Palabras clave}

Estudios; Comunicación; EEES; España; Grado; Master; Doctorado; Publicidad; Relaciones públicas; Comunicación audiovisual; Periodismo; Profesorado; Brecha de género; Universidades. 


\begin{abstract}
Communication studies in Spain have now become consolidated based on their evolution over the last 50 years. The aim of this paper is to provide an in-depth analysis of the current state of Spanish higher education in Communication. To this end, an analysis is carried out of the structure and composition of the undergraduate and postgraduate degrees (master and PhD studies) in Communication at public and private universities in Spain, paying special attention to the centers that host these studies, the people who contribute to their operation, and their offerings and distribution. Using a descriptive methodology and the content analysis technique, an analysis form is applied to the universities composing the system of Communication studies in Spain, based on their offerings and the information available and accessible on their institutional websites. The results reveal the difficulty of accessing such data and the lack of common criteria (or compliance) for presenting such information on official websites. The results reveal a greater offering at the national level but a wide dispersion of the higher education offering in terms of type, location, type of university, and territorial distribution. We also highlight the gender gap in terms of faculty leadership as well as among teaching staff. We emphasize the phenomenon of double or combined degrees, especially at private universities.
\end{abstract}

\title{
Keywords
}

Studies; Communication; EHEA; Spain; Bachelor; Master; PhD; Advertising; Public relations; Audiovisual communication; Journalism; Teaching staff; Gender; Universities.

\section{Introducción y estado de la cuestión: surgimiento y evolución de los estudios universitarios en Comunicación en España}

Con la creación de las primeras facultades de Ciencias de la Información en 1971 se inicia la andadura universitaria de los estudios de Comunicación en España. En el preámbulo del Decreto del Ministerio de Educación y Ciencia n. 2070/1971 de 13 de agosto (España, 1971), se establece que es conveniente

"insertar los estudios que se refieren al campo de los medios de comunicación social en la nueva estructura universitaria e incorporarlos a la dinámica general de los procesos educativos".

Y en su artículo segundo se señala que la impartición de estas enseñanzas se referirá al "Periodismo, Cinematografía, Televisión, Radiodifusión y Publicidad". Para ello se crean tres secciones o ramas como son "Departamento de Periodismo, Departamento de las Ciencias de la Imagen Visual y Auditiva, y Departamento de Publicidad". Estos estudios se comienzan a impartir en la Universidad Complutense de Madrid, la Universitat Autònoma de Barcelona y la Universidad de Navarra (primera privada).

A lo largo de estos 50 años de existencia universitaria de los estudios de Comunicación, se han multiplicado las facultades, se ha creado un cuerpo de docentes y han pasado miles de estudiantes de grado, master y doctorado por estos centros. Los estudios de la Comunicación cuentan desde hace décadas con una alta demanda de formación (Aneca, 2005, p. 15) y sus respectivos centros tienen una ocupación de plazas ofertadas muy alta, por lo que han ido adquiriendo una significación universitaria relevante, al mismo tiempo que social, económica y política.

Cuando se realiza una revisión del papel de los estudios, la perspectiva profesional ha ido dejando paso, sin decaer, a aspectos más educativos como son la investigación e impacto transformador de los estudios para los futuros profesionales, pero también de la Comunicación como dimensión transcendental en la vida en sociedad (De-Moragas, 1988; Caffarel-Serra; Domínguez; Romano, 1989; Castillo-Esparcia; Carretón-Ballester, 2010; Repiso; Torres-Salinas, 2014).

El marco regulatorio no ha sido un condicionante menor en esta trayectoria. Las sucesivas leyes universitarias en Europa y también en España como son la Ley orgánica 11/1983 de reforma universitaria (LRU) (España, 1983), la Ley orgánica 6/2001 de universidades (LOU) (España, 2001) y la Ley orgánica 4/2007 de modificación de la LOU (España, 2007a) han ido modulando la estructura de los estudios universitarios y han sido observadoras de la expansión de los estudios de Comunicación por toda España mostrando un panorama de presencia masiva en las universidades.

\section{MINISTERIO DE EDUCACION Y CIENCIA}

DECKETO $2070: 1971$, de 13 de agosto, por el que se regulan los estudios de. Periodismo $y$ dencis ni:dios de comunicacion somial en la Universidad.

Figura 1. Encabezado del decreto regulatorio de los estudios de Periodismo y demás medios de comunicación social en la universidad española. Fuente: Decreto 2070/1971, de 13 de agosto (España, 1971).

En este contexto, las investigaciones sobre el papel de la Comunicación han incidido en una evolución positiva de los estudios (De-Aguilera-Moyano, 1988; Gifreu, 1989; Castillo-Esparcia; Xifra, 2006; Repiso; Torres-Salinas; Delgado-López-Cózar, 2016). La producción sobre el rol de los estudios en Comunicación que se ha llevado a cabo en las facultades constata una creciente preparación en los futuros teóricos y en los análisis de la realidad social en varios enfoques: 
- Un estudio de las temáticas y de los objetos de estudio (Jones, 1998; Caffarel-Serra; Ortega-Mohedano; Gaitán-Moya, 2017; 2018; Martínez-Nicolás; Saperas-Lapiedra; Carrasco-Campos, 2017; 2018; Castillo-Esparcia; Carretón-Ballester; Pineda-Martínez, 2020).

- Aportaciones de las diferentes disciplinas al papel de la Comunicación (Martínez-Nicolás; Saperas-Lapiedra 2011; Lozano-Ascencio et al., 2020).

- El contexto institucional en el que se desarrollan los estudios (Saperas-Lapiedra 2016; Almansa-Martínez; Fernández-Souto, 2020; Martínez-Nicolás, 2020).

- Los interesantes debates y análisis sobre los desafíos y obstáculos que ha implicado la implementación del Espacio Europeo de Educación Superior (EEES) en el campo de la Comunicación (Marta-Lazo, 2009). En este sentido, en 2009 un monográfico de la revista Ícono 14, coordinado por Marta-Lazo propone una primera y necesaria revisión de las dimensiones y prácticas que por el momento estaba implicando el proceso de reconversión de la Comunicación en EEES en cuanto a: la formación propiamente dicha; la innovación docente y el uso de las TICs; aspectos legales y procesos adaptativos; implantación de los modelos basados en competencias; repercusiones sociales y políticas del cambio al nuevo sistema, entre otros.

El antecedente de la Declaración de Bolonia (1999) es la Declaración de La Sorbona (1998) en la que representantes de cuatro de los principales países europeos (Alemania, Francia, Italia y Reino Unido) plantearon y discutieron la necesidad de converger en un espacio común de Educación Superior para los estudios de grado y posgrado (masters "oficiales" o también denominados "universitarios" y doctorado). En posteriores encuentros se fue evaluando y revisando su implantación, al tiempo que se establecieron nuevos objetivos (Declaración de Praga, 2001; Declaración de Berlín, 2003; Declaración de Bergen, 2005; Declaración de Londres, 2007; Declaración de Lovaina, 2009 y Declaración de Budapest-Viena, 2010). Este proceso de convergencia de la enseñanza superior ha supuesto una modificación de la normativa que regula los estudios superiores en los países europeos (Ariza et al., 2012, p. 1010). En este sentido, la Declaración de Berlín (2003) plantea la necesidad de que el EEES incluya también los estudios de doctorado como última etapa en el proceso de enseñanza superior y primera en la formación de investigadores. La Declaración de Praga (2005) y el proyecto Doctoral Programmes for the European Knowledge Society promovido por la European University Association, diferencian claramente entre master y doctorado, cuando hablamos de estudios de posgrado. Esta declaración recalca que la enseñanza universitaria debe desempeñar un papel esencial en las sociedades contemporáneas, contribuyendo al desarrollo de los ámbitos económico, social y cultural. En este contexto, el doctorado se configura como el factor que debe coadyuvar en la mejora de la I+D+i, al considerar a los estudiantes de doctorado como investigadores en formación que, gracias a los resultados de sus proyectos de doctorado, impulsan el nuevo sistema. En el trabajo de Castillo-Esparcia, Álvarez-Nobell y Ruiz-Mora (2015, p. 717) se concluye que la reconfiguración de los estudios de doctorado en España representada por los reales decretos $R D$ 99/2011 (España, 2011) y RD 1393/2007 (España, 2007b), ha supuesto que

"Ios requisitos establecidos por la normativa para la aprobación de un programa de doctorado, han producido una reducción considerable de la oferta de doctorado"

e igualmente, ha significado la irrupción de las universidades privadas en la oferta de master y doctorado (Castillo-Esparcia; Álvarez-Nobell; Muñiz-Velázquez, 2013, p. 109).

El Libro blanco de los estudios de Comunicación (Aneca, 2005) buscaba realizar una propuesta que respondiese a la convergencia europea, que serviría de guía para los planes venideros (se plantea como una reestructuración) y siguiendo, especialmente, las pautas de las ya mencionadas declaraciones de Sorbona (1998), Bolonia (1999) y el comunicado de Praga (2001). En el estudio se constataba la presencia predominante en la universidad pública, pero se remarcaba que las universidades privadas estaban "adquiriendo una relevancia notable sobre todo en los últimos años" (Aneca, 2005, p. 105). Las comunidades de Cataluña (11) y Madrid (8) estaban a la cabeza en la titularidad de los centros que imparten nuestros estudios y en seis comunidades no existían los estudios de Comunicación: Aragón, Asturias, Baleares, Cantabria, Castilla-La Mancha y La Rioja (Aneca, 2005, p. 106). En la mayoría de las comunidades donde existían los estudios se impartían las tres titulaciones tradicionales: Comunicación Audiovisual, Periodismo y Publicidad y Relaciones Públicas. El $60 \%$ de las universidades son públicas y el $40 \%$ privadas (Aneca, 2005, p. 110); y en un $33 \%$ se contaba con facultades específicas para los estudios de Comunicación (Aneca, 2005, p. 111). En 2002, el número total de matriculados en los estudios fue de 33.000 estudiantes (Aneca, 2005, p. 113).

Igualmente relevante es el rol que la mujer ha desempeñado en este proceso. Los estudios de Comunicación han evolucionado y al igual que la sociedad, la mujer juega un papel clave en su desempeño. Martín-Algarra, Serrano-Puche y Rebolledo (2018, p. 81) en su estudio "La mujer en la investigación en comunicación en España: un análisis de la producción científica (2007-2013)", profundizan en la actividad investigadora de las mujeres en nuestro país, concretamente en la producción de tesis doctorales y el liderazgo en proyectos I+D financiados por convocatorias estatales. Los autores destacan que mientras en las tesis defendidas no hay diferencias entre hombres y mujeres (en el período de estudio), la mayor desigualdad se observa en el liderazgo de proyectos, donde el 69,9\% corresponde a hombres, frente al 30,1\% de mujeres. Destaca, además, que el $86,5 \%$ de la producción científica de las mujeres (tesis y proyectos) proviene de universidades públicas (Martín-Algarra; Serrano-Puche; Rebolledo, 2018, p. 78). 
Los datos del informe del Consejo Superior de Investigaciones Científicas sobre Mujeres Investigadoras 2021 (CSIC, 2021), corroboran en la actualidad la información anterior, aunque bien es cierto que este trabajo estudia las grandes áreas de conocimiento y no profundiza en las disciplinas de la Comunicación. Por escalas laborales, a nivel nacional y en el área de Humanidades y Ciencias Sociales (CSIC, 2021, p. 17), hay más científicos titulares hombres (56,1\%) que mujeres $(43,9 \%)$ y más profesores científicos $(71,8 \%)$ que profesoras $(28,2 \%)$; lo mismo ocurre con los investigadores (64,3\% son hombres y 35,7\%, mujeres). En Humanidades y Ciencias Sociales, solo el 34,2\% (CSIC, 2021, p. 26) de los proyectos europeos están liderados por una mujer y un 36,8\% (CSIC, 2021, p. 23) de los proyectos en convocatorias nacionales (datos 2020). En la misma área, donde se incluirían los estudios de Comunicación, el 60\% de las tesis son defendidas por mujeres (CSIC, 2021, p. 22).

\section{Metodología}

En este trabajo buscamos conocer el estado de los estudios de Comunicación de grado y posgrado (masters oficiales y doctorado) del sistema de educación superior en España, lo que permitirá ofrecer una radiografía del caso en cuestión a 50 años de su instauración. Para ello, nos planteamos las siguientes preguntas de investigación:

RQ1: ¿Qué características presenta la oferta actual de estudios universitarios oficiales en Comunicación en España? RQ2: ¿Existen diferencias entre la oferta de la universidad pública y la universidad privada?

RQ3: ¿Cómo se distribuyen geográficamente los títulos de Comunicación?

RQ4: ¿Qué caracteriza a los centros encargados de impartir dichos títulos?

RQ5: ¿Cuál es la presencia de la mujer en la enseñanza superior de Comunicación en España?

Para poder responder a las preguntas planteadas diseñamos una metodología de corte descriptivo que nos permitirá acotar, ordenar, caracterizar y clasificar el fenómeno objeto de estudio de forma precisa y exacta (Tinto-Arandes, 2013, p. 138). La técnica de investigación seleccionada es el análisis de contenido ya que, citando a Berelson (1952, p. 18), supone una técnica

"para la descripción objetiva, sistemática y cuantitativa de los contenidos manifiestos de la comunicación" y, además,

"es un método para la observación y la medición" (Kerlinger, 1973, p. 525).

La población objeto de estudio está compuesta por todas las universidades españolas integrantes del sistema de educación superior que imparten estudios oficiales de Comunicación de grado y posgrado (master y doctorado). Para acceder al listado de universidades se utiliza como filtro el buscador del Ministerio de Ciencia, Innovación y Universidades, "Quedu".

https://www.educacion.gob.es/notasdecorte/insercionLaboral.action

Las unidades de análisis están compuestas por las facultades (centros) y la oferta de grado, master y doctorado de cada universidad. El acceso a la información se realiza a través de las webs institucionales de los centros a partir de la oferta e información disponible y accesible. Para la codificación de los datos, se diseña una ficha de análisis con las siguientes variables:

1. Centros: tipo de universidad (pública/privada); ubicación geográfica; denominación; responsable; equipos directivos; denominación; género del profesorado.

2. Titulaciones: tipo (grado, doble grado/combinado, master oficial, doctorado); titularidad (pública/privada); ubicación geográfica; denominación y plazas ofertadas.

Inicialmente se preveía incluir también variables como tasa de graduación, líneas de investigación, tipo de profesorado y características de los coordinadores y responsables de las titulaciones. Pero estos datos no fueron fácilmente detectados de forma homogénea y completa durante la fase previa a la aplicación de la ficha, en la que se realizó un pretest con 15 títulos (5 de cada tipo) con el fin de identificar interferencias en el registro, realizar ajustes e introducir mejoras. Los datos se recogieron en el período abril-junio 2021 y se procesaron mediante hoja de cálculo y SPSS. ES necesario recalcar la ausencia de homogeneidad en las universidades a la hora de presentar la información básica y de cumplir con los criterios mínimos de información pública, lo que ha dificultado la recogida y procesamiento de los datos. La mayor complejidad se ha presentado en la ausencia de datos de plazas ofertadas (sobre todo en los títulos compuestos o los masters oficiales); los directorios de profesorado y la trazabilidad entre las titulaciones y los centros o departamentos responsables (sobre todo, en universidades privadas).

\section{Resultados}

\subsection{Estudios de grado y master oficial}

Los datos de los grados de Comunicación (tabla 1), muestran que en la actualidad existe en España una oferta de 161 grados, de ellos 90 se imparten en universidades públicas y 71 en universidades privadas. Geográficamente (tablas 2 y 3 ), la comunidad autónoma con una mayor oferta (tanto de plazas como de títulos) es Madrid, seguida de Cataluña, Andalucía y Comunidad Valenciana. En el puesto extremo encontramos a Baleares. En cuanto a la titularidad de las universidades 
(tabla 4), 38 son universidades públicas y 34 privadas. Las comunidades con un mayor número de universidades privadas son Madrid y Cataluña. Madrid cuenta con más universidades de titularidad privada que pública.

Tabla 1. Titulaciones y oferta según tipo de universidad en España

\begin{tabular}{|c|c|c|c|c|}
\hline \multirow{2}{*}{\multicolumn{2}{|c|}{ Perfil }} & \multicolumn{3}{|c|}{ Tipo de universidad } \\
\hline & & \multirow{2}{*}{$\begin{array}{c}\text { Públicas } \\
26\end{array}$} & \multirow{2}{*}{$\begin{array}{c}\text { Privadas } \\
70\end{array}$} & \multirow{2}{*}{$\begin{array}{r}\text { Total } \\
96\end{array}$} \\
\hline \multirow{3}{*}{ Titulaciones } & Doble grado / Grados combinados & & & \\
\hline & Grado & 71 & 90 & 161 \\
\hline & Master oficial & 65 & 49 & 114 \\
\hline \multirow{3}{*}{ Oferta de plazas } & Doble grado / Grados combinados & 845 & 4.256 & 5.101 \\
\hline & Grado & 7.244 & 8.318 & 15.562 \\
\hline & Master oficial & 2.244 & 4.573 & 6.817 \\
\hline
\end{tabular}

Tabla 2. Plazas ofertadas por Comunidades Autónomas y tipo de universidad

\begin{tabular}{|c|c|c|c|c|c|c|c|c|c|}
\hline \multirow{2}{*}{$\begin{array}{l}\text { Comunidad } \\
\text { autónoma }\end{array}$} & \multicolumn{3}{|c|}{ Universidades públicas } & \multicolumn{3}{|c|}{ Universidades privadas } & \multicolumn{3}{|c|}{ Totales } \\
\hline & DG/GC & Grado & Master & DG/GC & Grado & Master & DG/GC & Grado & Master \\
\hline Andalucía & 63 & 1.050 & 395 & 132 & 232 & & 195 & 1.282 & 395 \\
\hline Aragón & & 60 & 25 & 80 & 200 & 30 & 80 & 260 & 55 \\
\hline Baleares & & & & & 40 & & 0 & 40 & 0 \\
\hline Canarias & & 80 & 25 & 50 & 143 & & 50 & 223 & 25 \\
\hline Castilla y León & 20 & 445 & 100 & 140 & 270 & & 160 & 715 & 100 \\
\hline Castilla-La Mancha & 72 & 140 & & & & & 72 & 140 & 0 \\
\hline Cataluña & 50 & 1.429 & 319 & 380 & 3.180 & 1.670 & 430 & 4.609 & 1.989 \\
\hline Extremadura & 50 & 80 & 20 & & & & 50 & 80 & 20 \\
\hline Galicia & 10 & 370 & 115 & & & & 10 & 370 & 115 \\
\hline La Rioja & & & & & 650 & 1.650 & & 650 & 1.650 \\
\hline Madrid & 535 & 1.995 & 860 & 2.039 & 2.813 & 1.028 & 2.574 & 4.808 & 1.888 \\
\hline Murcia & & 280 & 30 & 360 & 180 & 65 & 360 & 460 & 95 \\
\hline Navarra & & & & 240 & 200 & 70 & 240 & 200 & 70 \\
\hline País Vasco & 30 & 320 & 65 & 50 & 125 & & 80 & 445 & 65 \\
\hline Valencia & 15 & 995 & 290 & 785 & 285 & 60 & 800 & 1.280 & 350 \\
\hline Total general & 845 & 7.244 & 2.244 & 4.256 & 8.318 & 4.573 & 5.101 & 15.562 & 6.817 \\
\hline
\end{tabular}

Si analizamos los números totales (del último año académico disponible en cada caso, recogidos en las tablas 1 y 2 ), encontramos que de forma nacional se ofertan 15.562 plazas de nuevo ingreso de grado, 8.318 en universidades públicas y 7.244 en universidades privadas. Por comunidades, Madrid y Cataluña son las que tienen una mayor oferta de plazas de grado, pero en ambos casos, la oferta de plazas privadas supera a las plazas públicas, siendo estos dos casos los únicos que rompen la tónica nacional. En tercer lugar y con números similares, Andalucía y la Comunidad Valenciana, donde la oferta pública es mayor a la privada. En el polo opuesto, encontramos a Baleares y Extremadura.

La oferta de dobles títulos/grados combinados es incipiente (96 títulos), representando el 37,3\% del total de grados (simples o compuestos) ofertados en España; mayoritariamente se ofertan en centros privados (70 títulos) aunque hay algunas iniciativas (26) en universidades públicas. En la comunidad autónoma de Madrid es donde se oferta un mayor número de estos estudios (43) con 2.039 plazas de titularidad privada y 535 públicas, es destacable que en este caso la oferta de dobles grados es muy similar a la de grado. A nivel nacional se ofertan 5.101 plazas, aproximadamente un tercio en comparación a la oferta de grado. Sin embargo, este dato presenta matices porque en muchos casos la información era ambigua, no estaba actualizada o estaba ausente.
Comparando los resultados de este estudio con los datos iniciales ofrecidos por el Libro blanco de los estudios de Comunicación del año 2005, encontramos que la oferta de estos estudios se ha incrementado, tanto en grado como en posgrado como desde el punto de vista geográfico. Lo que ha posibilitado y posibilitará un mayor acceso de la población española a estos estudios 
Tabla 3. Títulos ofertados por comunidades autónomas y tipo de universidad

\begin{tabular}{|c|c|c|c|c|c|c|c|c|c|}
\hline \multirow{2}{*}{ Comunidad autónoma } & \multicolumn{3}{|c|}{ Universidades públicas } & \multicolumn{3}{|c|}{ Universidades privadas } & \multicolumn{3}{|c|}{ Totales } \\
\hline & DG/GC & Grado & Master & DG/GC & Grado & Master & DG/GC & Grado & Master \\
\hline Andalucía & 3 & 8 & 12 & 3 & 5 & & 6 & 13 & 12 \\
\hline Aragón & & 1 & 1 & 2 & 4 & 1 & 2 & 5 & 2 \\
\hline Baleares & & & & & 1 & & 0 & 1 & 0 \\
\hline Canarias & & 1 & 1 & 1 & 3 & & 1 & 4 & 1 \\
\hline Cantabria & & & & 1 & 3 & 1 & 1 & 3 & 1 \\
\hline Castilla y León & 1 & 5 & 3 & 4 & 9 & & 5 & 14 & 3 \\
\hline Castilla-La mancha & 1 & 2 & & & & & 1 & 2 & 0 \\
\hline Cataluña & 2 & 19 & 11 & 6 & 19 & 13 & 8 & 38 & 24 \\
\hline Extremadura & 3 & 2 & 1 & & & & 3 & 2 & 1 \\
\hline Galicia & 1 & 6 & 4 & & & & 1 & 6 & 4 \\
\hline La Rioja & & & & & 2 & 6 & 0 & 2 & 6 \\
\hline Madrid & 12 & 10 & 18 & 31 & 33 & 21 & 43 & 43 & 39 \\
\hline Murcia & & 4 & 1 & 6 & 3 & 2 & 6 & 7 & 3 \\
\hline Navarra & & & & 3 & 2 & 3 & 3 & 2 & 3 \\
\hline País Vasco & 2 & 3 & 3 & 1 & 2 & & 3 & 5 & 3 \\
\hline Valencia & 1 & 10 & 10 & 12 & 4 & 2 & 13 & 14 & 12 \\
\hline Total general & 26 & 71 & 65 & 70 & 90 & 49 & 96 & 161 & 114 \\
\hline
\end{tabular}

La denominación de los títulos (tablas 5 y 6 ) presenta una doble vertiente. Por un lado, siguen vigentes los títulos tradicionales (Comunicación Audiovisual, Publicidad y Relaciones Públicas, Periodismo), pero se imponen nuevas denominaciones, influidas por los dobles títulos y la inclusión de las universidades privadas.

Con respecto a los estudios de master en Comunicación (tablas 1, 2 y 3), la oferta nacional total es de 114 masters, de ellos 65 títulos se imparten en universidades públicas y 49 en universidades privadas. Las plazas ofertadas de master ascienden a 6.817, 2.244 (33\%) para universidades públicas y 4.573 (67\%) para privadas. Como se observa, las universidades privadas cuentan con una oferta menor de títulos, pero estos están masificados. Sin embargo, este dato presenta matices porque en muchos casos el mismo era variable, no estaba actualizado o con frecuencia, estaba ausente.

Geográficamente la comunidad autónoma con una mayor oferta de masters es Cataluña (con 1.989 plazas y donde el $84 \%$ corresponde a plazas de universidades privadas), seguida de Madrid (1.880 total y $55 \%$ para la universidad privada) y La Rioja (1.650 plazas), en esta última todas las plazas son privadas y corresponden a una única universidad, la Universidad Internacional de La Rioja (UNIR). En los siguientes puestos estarían Andalucía, con 395 plazas ofertadas, y Comunidad Valenciana con 350 plazas. En el extremo encontramos a Extremadura con 1 master y 20 plazas. Se observa una amplia dispersión en la denominación de los masters, sin observarse un comportamiento homogéneo en esta variable.
Tabla 4. Tipo de universidad por comunidad autónoma

\begin{tabular}{|c|c|c|c|}
\hline $\begin{array}{l}\text { Comunidad } \\
\text { autónoma }\end{array}$ & $\begin{array}{c}\text { Universidad } \\
\text { pública }\end{array}$ & $\begin{array}{c}\text { Universidad } \\
\text { privada }\end{array}$ & Total \\
\hline Andalucía & 5 & 2 & 7 \\
\hline Aragón & 1 & 1 & 2 \\
\hline Baleares & & 1 & 1 \\
\hline Canarias & 1 & 2 & 3 \\
\hline Cantabria & & 1 & 1 \\
\hline Castilla La Mancha & 1 & & 1 \\
\hline Castilla y León & 4 & 4 & 8 \\
\hline Cataluña & 10 & 5 & 15 \\
\hline Extremadura & 1 & & 1 \\
\hline Galicia & 3 & & 3 \\
\hline La Rioja & & 1 & 1 \\
\hline Madrid & 5 & 10 & 15 \\
\hline Murcia & 1 & 1 & 2 \\
\hline Navarra & & 1 & 1 \\
\hline País Vasco & 1 & 2 & 3 \\
\hline Valencia & 5 & 3 & 8 \\
\hline Total general & 38 & 34 & 72 \\
\hline
\end{tabular}

Tabla 5. Denominaciones más frecuentes de los grados

\begin{tabular}{|l|c|}
\hline \multicolumn{1}{|c|}{ Denominaciones con mayor frecuencia } & Grados \\
\hline Comunicación Audiovisual & 42 \\
\hline Periodismo & 38 \\
\hline Publicidad y Relaciones Públicas & 30 \\
\hline Comunicación & 5 \\
\hline Publicidad & 3 \\
\hline Marketing y Comunicación Digital & 2 \\
\hline Periodismo y Comunicación Corporativa & 2 \\
\hline Comunicación Publicitaria & 2 \\
\hline Protocolo y Organización de Eventos & 2 \\
\hline Comunicación Digital & 2 \\
\hline
\end{tabular}


Tabla 6. Denominaciones más frecuentes de los dobles grados/grados combinados

\begin{tabular}{|l|c|}
\hline Denominaciones con mayor frecuencia & Doble grado/Grados combinados \\
\hline Periodismo + Comunicación Audiovisual & 21 \\
\hline Comunicación Audiovisual + Publicidad y Relaciones Públicas & 7 \\
\hline Periodismo + Publicidad y Relaciones Públicas & 7 \\
\hline Publicidad y Relaciones públicas + Marketing & 4 \\
\hline Periodismo + Relaciones Internacionales & 3 \\
\hline Periodismo + Historia & 3 \\
\hline Periodismo + Humanidades & 2 \\
\hline
\end{tabular}

\subsection{Programas de doctorado en España}

En el panorama español de programas de doctorado en Comunicación (tabla 7), la oferta total es de 34 entre universidades públicas y privadas. A su vez se realiza una oferta de más de 516 plazas para realizar estudios de doctorado. Finalmente, el profesorado que participa asciende a 744 docentes.

De estos 34 programas, 26 son ofertados por universidades públicas y 8 por privadas. Es necesario señalar que la configuración del real decreto

Tabla 7. Estudios de posgrado en España

\begin{tabular}{|l|c|c|c|}
\cline { 2 - 4 } \multicolumn{1}{c|}{} & $\begin{array}{c}\text { Universidades } \\
\text { públicas }\end{array}$ & $\begin{array}{c}\text { Universidades } \\
\text { privadas }\end{array}$ & Totales \\
\hline Programas & 26 & 8 & 34 \\
\hline Plazas & 428 & 88 & 516 \\
\hline Profesorado & 655 & 89 & 744 \\
\hline Hombres & 328 & 39 & 367 \\
\hline Mujeres & 327 & 50 & 377 \\
\hline
\end{tabular}

gramas de doctorado que planteaba exigencias como que el profesorado dispusiera de reconocimiento investigador, y en el caso de las públicas se refería a que el profesorado participante tuviera un alto porcentaje sexenios de investigación reconocidos. También aparece la exigencia de que es necesario que todas las líneas ofertadas en los programas de doctorado dispongan de proyectos de investigación que las sustenten y que esos proyectos sean espacios a través de los cuales los doctorandos puedan realizar sus tesis doctorales con una alta capacidad de finalizarlas, puesto que los proyectos permiten que las investigaciones estén mejor dirigidas y que se disponga de financiación para poder realizarlas. Otro elemento significativo es que se establece un límite temporal para poder realizar las tesis doctorales y que, pasado ese límite, el estudiante que no haya presentado su tesis deba causar baja en el programa de doctorado. Para ello se configuran estudiantes a tiempo completo que deben realizar su tesis doctoral en un período de 3 años y estudiantes a tiempo parcial que deben realizarla en un período ideal de 5 años. Para ambas modalidades de dedicación del estudiantado se permite una o como máximo dos prórrogas anuales, para la defensa de la tesis doctoral. Y, finalmente, un elemento de seguimiento y de control sobre los programas de doctorado son las evaluaciones anuales, que deben cumplir todos los estudiantes de doctorado, por parte de una comisión del programa de doctorado, en la que se supervisa que la propuesta y el cronograma de la tesis doctoral se vaya cumpliendo según lo establecido en la memoria inicial.

Por otro lado, un elemento que ha condicionado sobremanera la defensa de tesis doctorales es la necesidad de que, previo a la defensa pública, los estudiantes hayan tenido publicaciones sobre su tesis. Esas publicaciones y su calidad han sido interpretadas por cada programa de doctorado y sería muy interesante analizar en una posterior investigación qué tipo de publicaciones se exigen (revistas o libros) y cómo se justifica esa calidad ya sea en términos de la editorial que publica el libro o la posición de la revista en determinados rankings internacionales. Esta última inserción de exigencia para la defensa de la tesis doctoral implica una profunda transformación de los doctorandos y una posible carrera académica e investigadora en universidades puesto que los procesos de acreditación para la figura inicial, como es la de ayudante doctor, supone un mínimo de publicaciones y que ese mínimo, prácticamente se cumple con los requisitos establecidos con los programas de doctorado. Sería muy interesante analizar en otras investigaciones cómo estas exigencias de doctorado van a aumentar al alza las exigencias del número y calidad de publicaciones en esas figuras iniciales de la carrera del profesorado en las universidades.

\subsubsection{Oferta de estudios}

Cuando se analizan las denominaciones de los programas de doctorado que ofertan las universidades públicas y privadas, se observa que el denominador común no es recurrir a un título que hable solo de Comunicación, sino que la mayoría se refiere a temas de Ciencias Sociales englobando diferentes disciplinas como puede ser la Educación, la Antropología, la Lingüística, la Biblioteconomía o la Literatura. Eso implica que la oferta de doctorado se
Las comunidades con mayor oferta (tanto de plazas como de títulos) son Madrid, Cataluña, Andalucía y Valencia. Las comunidades con un mayor número de universidades privadas son Madrid y Cataluña; Madrid cuenta con más universidades de titularidad privada que pública 
ha centrado más en áreas que consideramos interdisciplinares. Es consecuencia de las exigencias del real decreto sobre un número mínimo de profesorado que sustente la oferta del programa y la necesidad de que cada una de las líneas ofertadas disponga de un proyecto de investigación financiado que avale esa investigación. Esos criterios han exigido que la estrategia para la creación de un programa de doctorado siga dos modelos:
La oferta de estudios se ha consolidado con 161 títulos de grado, 96 títulos de doble grado, 113 títulos de máster y 34 programas de doctorado. Igualmente, se constata un aumento de la oferta de los títulos en las universidades privadas

1. Con una perspectiva interdisciplinar, en el que se incluye una línea de comunicación que engloba tanto al Periodismo como la Comunicación Audiovisual o la Publicidad y las Relaciones Públicas. Esta estructura que incluye a diferentes disciplinas científicas es la que, en mayor medida, se produce en los programas de doctorado analizados en esta investigación. Su presencia es menor en las universidades públicas que en las privadas: en estas últimas, de los ocho programas de doctorado que se ofertan, solo uno recoge la denominación de doctorado en Comunicación Social. Se trata de Ceindo, un programa de doctorado de las universidades del CEU que permite que profesorado de dichas universidades pueda integrarse en un único programa de doctorado. También las universidades públicas ofrecen programas de doctorado interdisciplinares, principalmente en las universidades que no tienen un volumen muy alto de profesorado que pueda sustentar un único programa de doctorado en Comunicación.

2. Por otro lado, hay programas de doctorado que se centran únicamente en una oferta de comunicación y que se dan en las universidades que disponen de un alto volumen de profesorado, como son los casos de la Complutense de Madrid y de la Autònoma de Barcelona. En estas dos universidades encontramos no solo un único programa de doctorado, sino que en el caso de la Universidad Complutense de Madrid se ofertan dos, que intentan abarcar las dos áreas mayoritarias de Comunicación como son Periodismo, y Comunicación Audiovisual y Publicidad. En la Universitat Autònoma de Barcelona se ofrecen cuatro programas de doctorado, lo que supone una amplia oferta sobre estos estudios. Cabe señalar que también en las universidades públicas se ha intentado generar un programa de doctorado específico en Comunicación, a través de acuerdos interuniversitarios. Son los casos de la Universitat de Barcelona y la Universidad de Zaragoza, que ofrecen un programa de doctorado que abarca la Comunicación, pero también la Biblioteconomía. Y también es el caso singular de las universidades andaluzas, que han creado el Doctorado Interuniversitario en Comunicación entre la Universidades de Cádiz, Huelva, Málaga y Sevilla. Es un programa de doctorado con un número alto de plazas para estudiantes (85 entre las cuatro universidades) y que reúne a investigadores de las cuatro universidades andaluzas (con un total de 70), conformando uno de los programas de doctorado en los que participa mayor número de profesorado y en los que se oferta mayor número de plazas.

\subsubsection{Líneas de investigación}

Se observa que hay grandes diferencias entre unas universidades y otras. En las universidades públicas es donde se produce una mayor oferta de líneas con una media de 6,57 líneas por programa de doctorado. La que mayor número de líneas ofrece es la Universidad Complutense de Madrid con 25 en el programa de doctorado de Periodismo para que los estudiantes se puedan acoger a una de ellas. Le sigue, en segundo lugar, el programa de doctorado Comunicación Audiovisual y Publicidad de la Universitat Autònoma de Barcelona con 13 líneas y, en tercer lugar, el programa de doctorado Medios, Comunicación y Cultura también de la Universitat de Barcelona, con 9 líneas de investigación. El número medio de líneas de las universidades privadas es de 3 y eso supone una concentración del profesorado en unos campos de conocimiento más reducidos.

La proposición de diferentes líneas de investigación puede deberse a dos factores:

- en el caso de los programas de doctorado que engloban a diferentes disciplinas de las Ciencias Sociales, las líneas son expresión de esos campos de conocimiento y generalmente, estos programas de doctorado no reúnen un número excesivo de líneas ya que el profesorado de Comunicación se suele integrar en una línea referida a la Comunicación;

- en el caso de las universidades públicas con un alto número de proposiciones de líneas de investigación, interpretamos que se refiere a necesidades específicas de grupos de profesorado, que en el proceso de creación de ese programa de doctorado han contemplado líneas de investigación muy especializadas y que suponen una segmentación muy alta del profesorado y del alumnado. Parte de esta situación está motivada por la existencia de profesorado con una larga trayectoria en un área específica de la Comunicación y que pretende mantener esa línea generando espacios singulares para la realización de tesis doctorales, pero también ocurre que las líneas están asociadas a proyectos de investigación de ese profesorado que integra la línea en el área específica en el que han conseguido financiación por parte de las instituciones públicas o privadas.

Las facultades de Comunicación en España están lideradas en su mayoría por hombres y las plantillas del profesorado están masculinizadas, tanto en universidades públicas como privadas 


\subsubsection{La mujer en los programas de doctorado}

Uno de los aspectos que se han analizado en los programas de doctorado es el género (tabla 8), tanto en la coordinación o dirección, como en los docentes. En este sentido, se observa que en los programas de universidades públicas hay una distribución equilibrada entre mujeres y hombres en la coordinación de programas de doctorado, el 50\% de la coordinación de programas recae en mujeres. En cambio, en las universidades privadas la proporción es del 37,5\%.

Los resultados, sobre todo en las universidades públicas, constatan la creciente presencia femenina en posgrado y su consecuente liderazgo.

Tabla 8. Profesorado en los programas de las universidades

\begin{tabular}{|l|c|c|c|c|}
\cline { 2 - 5 } \multicolumn{1}{c|}{} & \multicolumn{2}{c|}{ Universidades públicas } & \multicolumn{2}{c|}{ Universidades privadas } \\
\cline { 2 - 5 } \multicolumn{1}{c|}{} & Hombres & Mujeres & Hombres & Mujeres \\
\hline Coordinación programas & 13 & 13 & 5 & 3 \\
\hline Profesorado & 328 & 327 & 39 & 50 \\
\hline
\end{tabular}

Se observa una distribución equitativa entre hombres y mujeres entre el profesorado de los programas de doctorado de las universidades públicas. Si atendemos a la presencia femenina en las universidades privadas, vemos que las mujeres son mayoría frente a los hombres como integrantes de la plantilla de doctores que participan en sus programas de doctorado.

\subsubsection{Oferta}

Conocer la oferta formativa de los programas de doctorado (tabla 7) permite discernir cuántas plazas proponen las universidades de los diferentes programas de doctorado. Así, los 34 que se ofrecen en España anualmente presentan 516 plazas. De estas, las de las universidades públicas son mayoritarias (428 plazas), lo que supone una media de 16,46 plazas por programa de doctorado. Por el contrario, las universidades privadas ofertan 88 plazas lo que supone una media de 11 personas por programa.

La presencia de la mujer en los programas de posgrado ha aumentado. En las universidades públicas hay una distribución equilibrada en la dirección de estos estudios, mientras que en las privadas solo el $37,5 \%$ de los programas son dirigidos por mujeres

Como es obvio existen diferencias entre universidades. El programa de doctorado que tiene mayor número de plazas es de la Universidad Rey Juan Carlos, si bien es cierto que es de Ciencias Sociales y no se especifica el número de plazas específicas para Comunicación. Si atendemos a programas cuya propuesta programática solo es de Comunicación, el programa interuniversitario entre las Universidades de Cádiz, Huelva, Málaga y Sevilla es el que ofrece mayor número de plazas de acceso con 85; en segundo lugar, aparece el programa de doctorado de Periodismo de la Universidad Complutense de Madrid con 65 plazas. En tercer lugar está un grupo amplio de universidades que ofrece 30 plazas en sus programas de doctorado.

\subsection{Centros y profesorado}

Un 58,4\% de los estudios de Comunicación (grado y posgrado) se ubican en centros que se dedican a impartir exclusivamente enseñanzas de Comunicación (tabla 9), mientras que un 41,6\% se combinan con otras disciplinas de las Ciencias Sociales. En su denominación, en 38 de los casos aparece la palabra "comunicación", frente a 27 que no lo incluyen.

Tabla 9. Especificidad disciplinar en la denominación de los centros

\begin{tabular}{|l|c|c|}
\hline Especificidad (denominación del centro) & Públicas & Privadas \\
\hline Sin mención del término "Comunicación & 15 & 12 \\
\hline Con mención del término "comunicación” & 23 & 15 \\
\hline Total general & $38(58,4 \%)$ & $27(41,6 \%)$ \\
\hline
\end{tabular}

En lo referente a la responsabilidad de los centros (tabla 10), 36 cargos son ostentados por hombres (decanos) y 22 mujeres (decanas), en 15 casos no se especifica. Por tipo de universidad no hay grandes diferencias. En cuanto a los equipos de gestión (tabla 11), en las universidades públicas hay poca diferencia entre los integrantes en materia de género (apenas 10 puntos), mientras que en las privadas son femeninos (91 mujeres y 62 hombres).
Tabla 10. Género de la dirección de los centros

\begin{tabular}{|l|c|c|c|}
\hline Denominación & Pública & Privada & Total general \\
\hline Decana & 14 & 8 & 22 \\
\hline Decano & 23 & 13 & 36 \\
\hline (en blanco) & 2 & 13 & 15 \\
\hline Total general & 39 & 34 & 73 \\
\hline
\end{tabular}


Los profesores que imparten docencia de grado y posgrado en España (tabla 12) son 4.149; de ellos, 2.314 trabajan en universidades públicas y 1.835 en privadas. Por género, los hombres predominan tanto en las universidades públicas como en las privadas y en porcentajes similares (por encima del 60\%).
Tabla 11. Equipos de gestión de los centros

\begin{tabular}{|l|c|c|}
\hline \multicolumn{1}{|c|}{ Tipo de universidad } & $\begin{array}{c}\text { Hombres en los } \\
\text { equipos }\end{array}$ & $\begin{array}{c}\text { Mujeres en los } \\
\text { equipos }\end{array}$ \\
\hline Pública & 163 & 154 \\
\hline Privada & 62 & 91 \\
\hline Total general & 225 & 245 \\
\hline
\end{tabular}

Tabla 12. Distribución del profesorado por género y tipo de universidad

\begin{tabular}{|c|c|c|c|}
\hline Profesorado (género) & Universidad pública & Universidad privada & Totales \\
\hline Masculino & $1.475(63,7 \%)$ & $1.229(67 \%)$ & 2.704 \\
\hline Femenino & $839(36,3 \%)$ & $606(33 \%)$ & 1.445 \\
\hline Subtotal & $2.314(100 \%)$ & $1.835(100 \%)$ & 4.149 \\
\hline
\end{tabular}

\section{Discusión y conclusiones}

Comparando los resultados de este estudio con los datos iniciales ofrecidos por el Libro blanco de los estudios de Comunicación del año 2005, encontramos que la oferta de estos estudios se ha incrementado, tanto en grado, como en posgrado, como desde el punto de vista geográfico. Esto ha posibilitado y posibilitará un mayor acceso de la población española a estos estudios. Geográficamente se sigue produciendo una conglomeración de los estudios en las comunidades de Cataluña y Madrid, que acaparan tanto un mayor número de títulos, como de plazas (tanto públicas como privadas). Aragón, Baleares, Cantabria, Castilla-La Mancha y La Rioja se han sumado a la oferta de estudios; en 2005 estas comunidades no tenían estudios en la materia que nos ocupa.

La oferta de estudios se ha consolidado con 161 títulos de grado, 96 títulos de doble grado o combinados, 114 títulos de master oficiales y 34 programas de doctorado. Igualmente, se constata un aumento de la oferta de los títulos en las universidades privadas, no solo con grados, sino con dobles grados o grados combinados, masters oficiales y programas de doctorado. El mayor número de centros privados se congregan en las comunidades de Madrid y Barcelona. En cuanto a las facultades o centros responsables, asistimos a una consolidación de instituciones específicas de Comunicación en contraprestación a los centros o facultades mixtas o interdisciplinares, en este caso se ha revertido la situación del año 2005.

El liderazgo de los centros es mayormente masculino, al igual que los integrantes de las plantillas, por lo que afirmamos que los cuerpos docentes de los estudios de Comunicación en España están masculinizados. Situación que se replica tanto en la universidad pública como en la privada. Por el contrario, esta investigación constata la creciente presencia femenina en los posgrados y su consecuente liderazgo de los programas, consecuencia de una actividad investigadora activa y sostenida por las mujeres en los últimos años, como constatan las investigaciones citadas. Aunque esta situación parece haberse instaurado en las universidades públicas, las universidades privadas aún necesitan mejorar en esta materia (en el liderazgo de programas de doctorado).

Por lo tanto, vemos que a pesar de ser menos como integrantes de las plantillas de los centros donde se imparten los estudios de Comunicación, las mujeres han realizado un gran esfuerzo por llegar a formar parte de los equipos de doctorado; no podemos obviar la importancia que tiene para una mujer (y su carrera investigadora) poder formar un equipo de trabajo que le permita poder presentar proyectos a las convocatorias públicas de I+D+i.

Los resultados demuestran que aún queda trabajo para poder alcanzar la igualdad efectiva y romper con el techo de cristal. Interpretamos estos resultados como un signo de alerta para reclamar un mayor compromiso con la igualdad y visibilidad de la mujer como agente activo en los estudios de Comunicación. Una mayor presencia de la mujer en las enseñanzas superiores contribuye a visibilizar su trabajo y rompe con los roles tradicionales de liderazgo y transmisión de conocimiento masculinos en el ecosistema social de la Comunicación. Será más fácil, por tanto, aumentar el número de profesoras e investigadoras en el EEES y en el sistema de I+D+i de nuestro país, si nuestras estudiantes trabajan día a día con mujeres que lideran centros, equipos, proyectos... con las que se puedan identificar.

Otra cuestión importante sobre la que debemos reflexionar es la accesibilidad a la información. Las universidades deben trabajar por mejorar la información que ofrecen en sus webs, como vía para relacionarse con sus destinatarios y como ejercicio de transparencia hacia la sociedad. Muchos de los centros estudiados ofrecen información que responde meramente a criterios de acreditación (principalmente, entre las universidades públicas) y parece que se limitan a cumplir un listado de tareas para superar con éxito el proceso de evaluación; otras, transforman sus webs en un folleto promocional que solo busca conseguir que los estudiantes rellenen un formulario (práctica más común entre las universidades privadas). Se hace necesario, por tanto, incluir criterios de accesibilidad, usabilidad y transparencia en el diseño y contenido de estos canales y convertir estos espacios en oportunidades para la rendición de cuentas en materia de resultados académicos, composición de las plantillas e información sobre los títulos.

Nos gustaría concluir proponiendo futuros estudios a partir de nuestro trabajo; estos podrían incidir en la tasa de éxito de los estudios analizados, las salidas profesionales y el grado de inserción laboral de los egresados. Igualmente, sería interesante conocer el grado de estabilidad laboral del profesorado, su antigüedad y formación. 
Como limitaciones de este estudio planteamos la dificultad experimentada por este equipo de trabajo en el acceso a la información y su procesamiento. Al mismo tiempo, planteamos la necesidad de realizar un análisis cruzado con los datos disponibles de la Agencia Nacional de Evaluación de la Calidad y la Acreditación, para comparar los resultados e inferir nuevas propuestas de investigación.

\section{Referencias}

Almansa-Martínez, Ana; Fernández-Souto, Ana-Belén (2020). "Professional public relations (PR) trends and challenges". Profesional de la información, v. 29, n. 3, e290303.

https://doi.org/10.3145/epi.2020.may.03

Aneca (2005). Libro blanco. Títulos de grado en comunicación. http://www.aneca.es/var/media/150336/libroblanco_comunicacion_def.pdf

Ariza, Tania; Bermúdez, M. Paz; Quevedo-Blasco, Raúl; Buela-Casal, Gualberto (2012). “Evolución de la legislación de doctorado en los países del EEES". Revista iberoamericana de psicología y salud, v. 3, n. 2, pp. 89-108.

http://www.ugr.es/ aepc/articulo/4.pdf

Berelson, Bernard (1952). Content analysis in communication research. NY: Hafner Press. ISBN: 0028412109

Caffarel-Serra, Carmen; Domínguez, Milagros; Romano, Vicente (1989). “El estado de la investigación de comunicación en España (1978-1987)”. Cuadernos de investigación en comunicación (Cinco), n. 3, pp. 45-57.

Caffarel-Serra, Carmen; Ortega-Mohedano, Félix; Gaitán-Moya, Juan-Antonio (2017). “Investigación en Comunicación en la universidad española en el período 2007-2014". El profesional de la información, v. 26, n. 2, pp. 218-227. https://doi.org/10.3145/epi.2017.mar.08

Caffarel-Serra, Carmen; Ortega-Mohedano, Félix; Gaitán-Moya, Juan-Antonio (2018). "Communication research in Spain: Weaknesses, threats, strengths and opportunities. [La investigación en comunicación en España: Debilidades, amenazas, fortalezas y oportunidades]". Comunicar, v. 26, n. 56, pp. 61-70.

https://doi.org/10.3916/C56-2018-06

Castillo-Esparcia, Antonio; Álvarez-Nobell, Alejandro; Muñiz-Velázquez, José-Antonio (2013). "EEES y la formación en comunicación en España. La estructura de la oferta de posgrado". Estudios sobre el mensaje periodístico, n. 19, pp. 99-110. https://doi.org/10.5209/rev_ESMP.2013.v19.42015

Castillo-Esparcia, Antonio; Álvarez-Nobell, Alejandro; Ruiz-Mora, Isabel (2015). "Procesos de institucionalización de la investigación sobre comunicación: la reconfiguración de la estructura de la oferta de los estudios de doctorado en comunicación en España". En: Díaz-Nosty, Bernardo; De-Frutos-García, Ruth (coords.). Rumbos de la investigación en España sobre comunicación. Málaga: Universidad de Málaga; AEIC, pp. 722-738. ISBN: 9788460870531

https://dialnet.unirioja.es/servlet/articulo?codigo=7340086

Castillo-Esparcia, Antonio; Carretón-Ballester, Carmen (2010). "Research in Communication. Bibliometric study in journals of Communication in Spain". Communication \& society, v. 23, n. 2, pp. 289-327.

https://revistas.unav.edu/index.php/communication-and-society/article/view/36234

Castillo-Esparcia, Antonio; Carretón-Ballester, Carmen; Pineda-Martínez, Paula (2020). “Investigación en relaciones públicas en España". Profesional de la información, v. 29, n. 3, e290330.

https://doi.org/10.3145/epi.2020.may.30

Castillo, Antonio; Xifra, Jordi (2006). “Investigación bibliométrica de las tesis doctorales españolas sobre relaciones públicas (1965-2005)". Análisi: quaderns de comunicació i cultura, n. 34, pp. 141-161.

https://raco.cat/index.php/Analisi/article/view/55449

CSIC (2021). Informe mujeres investigadoras. Comisión de mujeres y ciencia del CSIC. https://www.csic.es/sites/www.csic.es/files/informe_mujeres_investigadores_cmyc-2021_0.pdf

De-Aguilera-Moyano, Miguel (1998). "La investigación sobre comunicación en España: una visión panorámica”. Comunicación y cultura, n. 4, pp. 5-11.

https://dialnet.unirioja.es/servlet/articulo?codigo $=2901273$

De-Moragas, Miquel (1988). “Los estudios sobre comunicación y nuevas tecnologías en España: indicaciones sobre sus antecedentes y estado actual”. Cuadernos de investigación en comunicación (Cinco), n. 1, pp. 11-19.

España (1971). “Decreto 2070/1971, de 13 de agosto, por el que se regulan los estudios de Periodismo y demás medios de comunicación social en la universidad". BOE, n. 220, 14 septiembre.

https://www.boe.es/diario_boe/txt.php?id=BOE-A-1971-1180

España (1983). “Ley orgánica 11/1983, de 25 de agosto, de reforma universitaria”. BOE, n. 209, 1 septiembre.

https://www.boe.es/buscar/doc.php?id=BOE-A-1983-23432 
España (2001). “Ley orgánica 6/2001, de 21 de diciembre, de universidades”. BOE, n. 307, 24 diciembre. https://www.boe.es/eli/es/lo/2001/12/21/6

España (2007a). “Ley orgánica 4/2007, de 12 de abril, por la que se modifica la Ley orgánica 6/2001, de 21 de diciembre, de universidades". BOE, n. 89, 13 abril.

https://www.boe.es/buscar/doc.php?id=BOE-A-2007-7786

España (2007b). "Real decreto 1393/2007, de 29 de octubre, por el que se establece la ordenación de las enseñanzas universitarias oficiales". BOE, n. 260, 31 octubre.

https://www.boe.es/buscar/act.php?id=BOE-A-2007-18770

España (2011). "Real decreto 99/2011, de 28 de enero, por el que se regulan las enseñanzas oficiales de doctorado". $B O E$, n. 35, 11 febrero.

https://www.boe.es/buscar/act.php?id=BOE-A-2011-2541

Gifreu, Josep (1989). "La investigació de la comunicació a Catalunya: assaig de periodització”. Anàlisi: quaderns de comunicació i cultura, n. 12, pp. 9-65.

https://www.raco.cat/index.php/Analisi/article/view/41077

Jones, Daniel E. (1998). “Investigación sobre comunicación en España: evolución y perspectivas”. Zer, v. 3, n. 5. https://ojs.ehu.eus/index.php/zer/article/view/17359

Kerlinger, Fred N. (1973). Foundations of behavioural research, $2^{\text {nd }}$ ed. NY: Holt, Rinehart and Winston. ISBN: 0030854628

Lozano-Ascencio, Carlos; Gaitán-Moya, Juan-Antonio; Caffarel-Serra, Carmen; Piñuel-Raigada, José-Luis (2020). “Una década de investigación universitaria sobre comunicación en España, 2007-2018”. Profesional de la información, v. 29 , n. 4, e290412. https://doi.org/10.3145/epi.2020.jul.12

Marta-Lazo, Carmen (2009). “El proceso de reconversión de la comunicación en el EEES: presentación”. Icono14, v. 7, n. 3. https://doi.org/10.7195/ri14.v7i3.492

Martín-Algarra, Manuel; Serrano-Puche, Javier; Rebolledo, Marta (2018). “La mujer en la investigación en comunicación en España: un análisis de la producción científica (2007-2013)”. AdComunica, n. 15, pp. 65-87.

https://doi.org/10.6035/2174-0992.2018.15.5

Martínez-Nicolás, Manuel (2020). "La investigación sobre comunicación en España (1985-2015). Contexto institucional, comunidad académica y producción científica". Revista latina de comunicación social, n. 75, pp. 383-414.

https://doi.org/10.4185/RLCS-2020-1432

Martínez-Nicolás, Manuel; Saperas-Lapiedra, Enric (2011). “La investigación sobre comunicación en España (1998-2007). Análisis de los artículos publicados en revistas científicas”. Revista latina de comunicación social, n. 66, pp. 101-129.

https://doi.org/10.4185/RLCS-66-2011-926-101-129

Martínez-Nicolás, Manuel; Saperas-Lapiedra, Enric; Carrasco-Campos, Ángel (2017). "Journalism research in Spain. Analysis of research articles published in Spanish journals over the past 25 years (1990-2014)". Communication \& society, v. 30, n. 4, pp. 149-166.

https://revistas.unav.edu/index.php/communication-and-society/article/view/35764

Martínez-Nicolás, Manuel; Saperas-Lapiedra, Enric; Carrasco-Campos, Ángel (2018). “La investigación sobre comunicación en España en los últimos 25 años (1990-2014). Objetos de estudio y métodos aplicados en los trabajos publicados en revistas españolas especializadas". Revista de metodología de ciencias sociales, n. 42, pp. 37-69.

https://doi.org/10.5944/empiria.42.2019.23250

Repiso, Rafael; Torres-Salinas, Daniel (2014). “Comunicación y documentación: dos disciplinas convergentes pero distintas". Anuario ThinkEPI, v. 8, pp. 225-229.

https://thinkepi.profesionaldelainformacion.com/index.php/ThinkEPI/article/view/29585

Repiso, Rafael; Torres-Salinas, Daniel; Delgado-López-Cózar, Emilio (2016). “Análisis de la relación entre disciplinas a través del uso de tesis doctorales. El caso de televisión, radio, cine y fotografía en España". Revista latina de comunicación social, v. 71, pp. 874-890.

https://doi.org/10.4185/RLCS-2016-1125

Saperas-Lapiedra, Enric (2016). “Cuatro décadas de investigación comunicativa en España. Los procesos de institucionalización y de profesionalización de la investigación (1971-2015)". Disertaciones. Anuario electrónico estudios de comunicación social, v. 9, n. 2, pp. 27-45.

https://doi.org/10.12804/disertaciones.09.02.2016.02

Tinto-Arandes, José-Antonio (2013). “El análisis de contenido como herramienta de utilidad para la realización de una investigación descriptiva. Un ejemplo de aplicación práctica utilizado para conocer las investigaciones realizadas sobre la imagen de marca de España y el efecto país de origen". Provincia, n. 29, pp. 135-173.

https://www.redalyc.org/articulo.oa?id $=55530465007$ 